\title{
Interfacial Engineering of Reduced Graphene Oxide for High-Performance
}

\section{Supercapacitor Materials}

Huilian Hao ${ }^{1 *}$, Jianjun Wang ${ }^{1,2}$, Qiu Lv, ${ }^{1}$ Yiding Jiao ${ }^{3}$, Jing Li ${ }^{1}$, Wenyao Li 1,3,7*, Isil Akpinar ${ }^{6,7}$, Wenzhong Shen ${ }^{5}$ and Guanjie $\mathrm{He}^{3,4 *}$

${ }^{1}$ School of Materials Engineering, Shanghai University of Engineering Science, 333 Long Teng Road, Shanghai 201620, China

${ }^{2}$ College of Chemistry, Beijing Normal University, No.19 Xinjiekouwai St., Beijing 100875, China

${ }^{3}$ Department of Chemistry, University College London, Christopher Ingold Laboratory, 20 Gordon Street, London WC1H 0AJ

${ }^{4}$ School of Chemistry, University of Lincoln, Joseph Banks Laboratories, Green Lane, Lincoln, LN6 7DL, U.K

${ }^{5}$ Institute of Solar Energy, and Key Laboratory of Artificial Structures and Quantum Control (Ministry of Education), Department of Physics and Astronomy, Shanghai Jiao Tong University, 800 Dong Chuan Road, Shanghai 200240, China

${ }^{6}$ Department of Environmental Engineering, Aksaray University, 68100 Aksaray, Turkey

7 Department of Chemical Engineering, University College London, Electrochmical Innovation Labortory, United Kingdom, WC1E 7JE, London

*Corresponding author: Huilian Hao (e-mail: sulee8866@126.com), Wenyao Li(email:liwenyao314@gmail.com), Guanjie He (e-mail:ghe@lincoln.ac.uk) 
Abstract: To solve the problems related to the re-stacking of reduced graphene oxides (rGO) and further improve their surface chemical behaviors to satisfy supercapacitor demands. The rGO decorated with graphene quantum dots has been successfully prepared via a facile lowpower ultrasonic method. It is demonstrated the graphene quantum dots/reduced graphene oxide electrode has a high specific capacitance of $312 \mathrm{~F} \mathrm{~g}^{-1}$, which is nearly three times higher than that of the reduced graphene oxide (132 $\left.\mathrm{F} \mathrm{g}^{-1}\right)$. The enhanced super-capacitive performances of graphene quantum dots/reduced graphene oxide have been attributed to the introduction of graphene quantum dots, which effectively prevent the aggregation and restacking of reduced graphene oxide sheets, promoting its surface exposed to the electrolyte for sufficient mass transfer. Meanwhile, these features provide more pathways for the transportation of electrons between the interlayer of reduced graphene oxide sheets. Afterward, a detailed energy storage mechanism was analyzed.

Keywords: Graphene quantum dots; Reduced graphene oxide; Synergistic effect; Asymmetric supercapacitors 


\section{Introduction}

In recent years, with the increasing demands for portable electronics and different microisilpower systems, supercapacitors have received tremendous attention owing to its fast charge/discharge, high power density, and long cycle life [1, 2]. Moreover, to satisfy the increasing energy demands, researchers are exploiting the advanced electrode materials. Graphene has been proposed as one of the most promising candidates, due to its exceptionally electrochemical stability and good electronic conductivity $[3,4]$. However, the agglomeration and re-stacking inevitably appear as a result of $\pi-\pi$ interactions of graphene, which decreases the surfaces that required for the electrochemical charge storage, leading to a low specific capacitance $\left(C_{s}\right)$ [5]. To address this problem, multiple graphene composites have been prepared for supercapacitors, such as reduced graphene oxide (rGO)/Au [6], $\mathrm{rGO} / \mathrm{MnO}_{\mathrm{x}}$ [7], rGO/MoS 2 [8], and N, S and P co-doped rGO composite electrodes [9]. Although these attempts obtained enhanced specific capacitance compared with pure graphene, the highly tortuous structures that endowed by the morphological effect on the scalability of graphene are destroyed, which weakens their rate capability and cycling stability.

Recently, it has been reported that graphene combined with other different dimensional carbon materials can efficiently improve the $C_{\mathrm{s}}$ through their synergistic effects $[10,11]$. Among these carbon structures, the graphene quantum dots (GQDs) have become a spotlight in the fundamental research due to their high good electrical conductivity and large specific surface area [12-15]. Moreover, GQDs employed as electrode materials usually exhibit strong hydrophilicity, which can improve the wettability of the surface, leading to a fast electron transfer [16, 17]. Although GQDs exhibit high specific capacitance when used alone as an electrode material [17], the materials synthesis and electrode fabrication process needs to be further improved. Therefore, the composites of rGO and GQDs are investigated accordingly, that can exploit both advantages of GQDs and rGO [18]. Recently, Samantara et al. have reported that $\mathrm{rGO} / \mathrm{CQDs}-\mathrm{HP}$ for supercapacitors with a $C_{\mathrm{s}}$ of $156 \mathrm{~F} \mathrm{~g}^{-1}$ at $0.13 \mathrm{~A} \mathrm{~g}^{-1}$ [19], 
Dang et al. have reported that the $\mathrm{CD} / \mathrm{rGO}$ has been prepared by the hydrogen dielectric barrier discharge plasma technique with a $C_{\mathrm{s}}$ of $211.9 \mathrm{~F} \mathrm{~g}^{-1}$ at $0.5 \mathrm{~A} \mathrm{~g}^{-1}$ [20]. Although they successfully prepared the rGO/GQDs composites and applied them as supercapacitor electrode materials, the synergistic effect between GQDs and rGO for the enhanced electrochemical performances needs to be further understood.

In this work, a low-power ultrasonic method has been employed to prepare rGO with GQDs on the surface; the ultrasonic process promotes uniform in situ adsorption of GQDs on the surface of rGO, which effectively prevents aggregation and restacking of rGO nanosheets. The rGO/GQDs (3:3) electrode for supercapacitor shows a $C_{s}$ of $312 \mathrm{~F} \mathrm{~g}^{-1}$ and a high $C_{\mathrm{s}}$ retention of $93 \%$ after 10,000 cycles. Meanwhile, the electrode exhibits good rate capability when the current densities increased from 0.5 to $10 \mathrm{~A} \mathrm{~g}^{-1}\left(81.4 \% C_{s}\right.$ retention with 20 times increase of current density). The rGO/GQDs//active carbon (AC) asymmetric supercapacitors (ACS) displays the energy density of $22.2 \mathrm{~W} \mathrm{~h} \mathrm{~kg}^{-1}$ at $500 \mathrm{~W} \mathrm{~kg}^{-1}$ with $C_{\mathrm{s}}$ retention of $91.6 \%$ after 10,000 cycles. Synergistic effect between GQDs and rGO for the enhanced electrochemical performances has been studied thoroughly by quantifying the relative contributions from capacitive behaviors $\left(C_{d l}\right)$ and diffusion-controlled $\left(C_{d}\right)$ processes. With the increase of $\mathrm{rGO} / \mathrm{GQD}$ mass ratio from 3:0 to $3: 3$, the $C_{d}$ contribution maintains a ratio of about $30 \%$, but the $C_{d l}$ increase significantly, which demonstrates that surface-modified GQDs can provide more active sites for rGO and optimize the structure of the rGO.

\section{Experimental Section}

\subsection{Chemicals}

Glucose, graphite powder, sulfuric acid, sodium hydroxide, AC, and hydrazine hydrate were purchased from Aladdin reagent. These reagents were used without further purification.

\subsection{Preparation of GQDs}


The GQDs were synthesized by a hydrothermal approach. In the procedure of GQDs preparation, $1.2 \mathrm{~g}$ of glucose and $8 \mathrm{ml}$ of $\mathrm{H}_{2} \mathrm{SO}_{4}$ were added into $12 \mathrm{ml}$ of deionized water. The mixture was treated in reaction kettle at $200{ }^{\circ} \mathrm{C}$ for $3 \mathrm{~h}$. The glucose molecules were dehydrated under hydrothermal conditions and then converted to GQDs, as shown in Scheme 1. Then, the sample was neutralized with sodium hydroxide and purified by a dialysis tube (1000 Da).

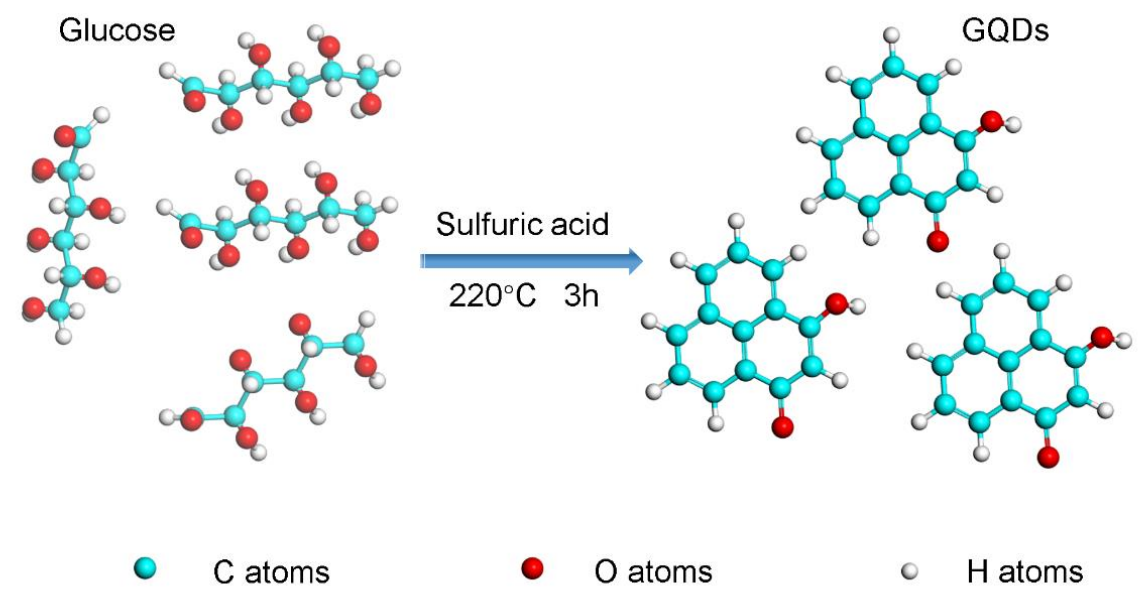

Scheme 1 Synthetic mechanism of GQDs.

\subsection{Preparation of rGO and rGO/GQDs}

Graphene oxide was synthesized by a modified Hummers approach [21]. The rGO was prepared by using the reductant of hydrazine hydrate [22]. $100 \mathrm{mg}$ of graphene oxide, $100 \mathrm{ml}$ of deionized water, and $1 \mathrm{ml}$ of hydrazine hydrate were added in a $250 \mathrm{~mL}$ round-bottom flask. This dispersion was sonicated in an ultrasonic bath cleaner $(100 \mathrm{~W})$, and then heated in a water bath at $95{ }^{\circ} \mathrm{C}$ for $10 \mathrm{~h}$. These products were washed by deionized water and methanol. Finally, rGO powder was obtained by freeze-drying the as-prepared samples.

The rGO with the surface modification of GQDs was prepared by a low-power ultrasonic method. $30 \mathrm{mg}$ of rGO was added to $30 \mathrm{~mL}$ of GQDs solution, the mixture was sonicated for $3 \mathrm{~h}$ and filtrated through a $20 \mathrm{~nm}$ anodic aluminum oxide membrane. The free GQDs were 
removed by re-dispersing and filtering the product. The rGO/GQDs composite was obtained by freeze-drying the above product. Four rGO/GQDs composite powders were prepared by changing the mass ratio of rGO and GQDs from 3:0 to 3:3.

\subsection{Characterization}

Scanning electron microscope (SEM, S-4800), Transmission electron microscope (TEM, JEM2100F) and atomic force microscope (AFM, NT-MDT Prima) were measured to characterize the structure of GQDs, rGO, and rGO/GQDs samples. Fourier transform infrared spectra (FTIR) were measured to find the chemical bonds of GQDs, rGO, and rGO/GQDs at Nicolet Nexus 870 FTIR spectrometer within 1000-4000 $\mathrm{cm}^{-1}$ range. Raman scattering spectra were performed to monitor the degree of structural order of GQDs on a Jobin Yvon Lab RAM HR spectrometer using $325 \mathrm{~nm}$ line of Ar ion laser. UV-visible spectra were performed by a Perkin-Elmer Lambda 20 spectrometer. Photoluminescence (PL) spectra were performed by Hitachi F-7000. X-ray photoelectron spectroscopy (XPS) spectra (ThermoFisher, ESCALAB $250 \mathrm{XI}$ ) was employed to analyze the contents of oxygen and carbon in $\mathrm{rGO}$ and rGO/GQDs. Nitrogen sorption measurements were carried out using an automatic volumetric sorption analyzer (ASAP 2020). Contact angle (CA) measurements were carried out by a JC2000D2A water contact angle tester.

\subsection{Electrochemical Measurements}

Electrodes were assembled by mixing rGO/GQDs, carbon black and polytetrafluoroethylene in a mass ratio of 8:1:1. It was coated on a flexible graphite foil $(1.0 \mathrm{~cm} \times 1.0 \mathrm{~cm})$ and dried at $60{ }^{\circ} \mathrm{C}$ for $6 \mathrm{~h}$; nearly $2 \mathrm{mg}$ of electroactive material was loaded on the current collectors. The ASC was assembled by coordinating $\mathrm{rGO} / \mathrm{GQDs}$ and $\mathrm{AC}$ as the positive and negative electrode, respectively. Galvanostatic charge/discharge (GCD), cyclic voltammetry (CV), and electrochemical impedance spectroscopy (EIS) were measured by the CHI-660E electrochemical workstation. 


\section{Results and Discussion}

\subsection{Microstructure Characterization}
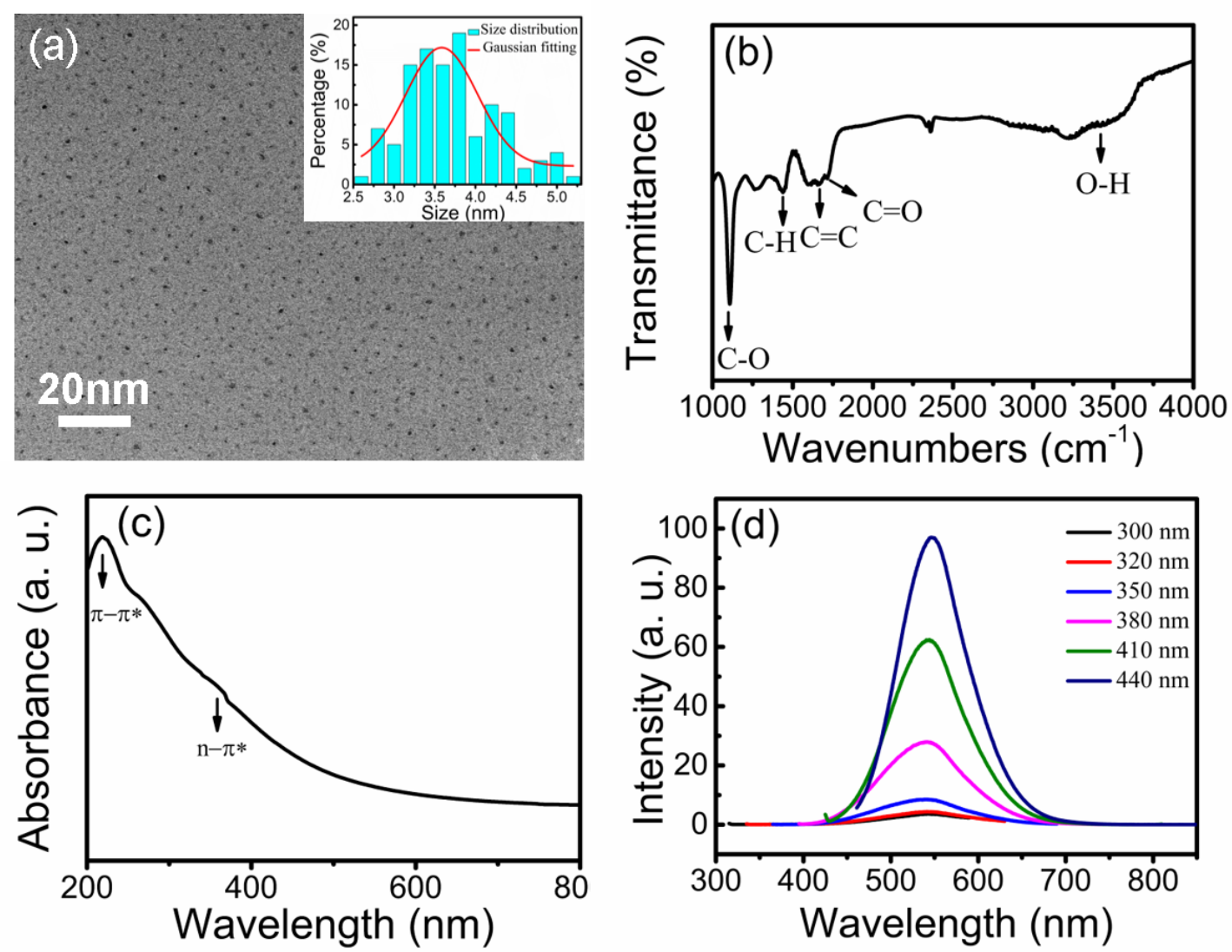

Figure 1 (a) TEM image of the GQDs, inset: size distribution of GQDs with the Gaussian fitting (red curve). (b) FTIR spectrum of GQDs. (c) UV-vis absorption spectrum and (d) PL spectra of GQDs.

Figure 1a shows the TEM image of the synthesized GQDs. It can be observed that GQDs with a lateral size distribution of 2.5-5.2 nm are well monodispersed. Statistical analysis of the GQDs in TEM image shows the average size of GQDs is $~ 3.6 \mathrm{~nm}$, as shown in the inset of Figure 1a. Figure 1b presents the FTIR spectrum of GQDs, the absorption peaks at 1103, $1654,1702,3430$ and $1432 \mathrm{~cm}^{-1}$ can be ascribed to the modes of $\mathrm{C}-\mathrm{O}, \mathrm{C}=\mathrm{C}, \mathrm{C}=\mathrm{O}, \mathrm{O}-\mathrm{H}$ stretching vibrations and $\mathrm{C}-\mathrm{H}$ bending vibration, respectively. The presence of $\mathrm{C}=\mathrm{C}$ bonds indicates that the ordered carbon backbone structure is formed in GQDs. The existence of C- 
$\mathrm{O}, \mathrm{C}=\mathrm{O}$ and $\mathrm{O}-\mathrm{H}$ demonstrates that GQDs contain the oxygen-containing groups and are incompletely carbonized [23-25]. Meanwhile, the presence of oxygen-containing groups facilitates electron transfer of GQDs electrode material for energy storage applications [16]. Figure S1 (Supporting Information) displays the Raman spectrum of GQDs. It can be seen that the D band at $1360 \mathrm{~cm}^{-1}$ is ascribed to structural defects, and $\mathrm{G}$ band at $1590 \mathrm{~cm}^{-1}$ is associated with the $s p^{2}$ carbon structure. The $\mathrm{ID}_{\mathrm{D}} / \mathrm{I}_{\mathrm{G}}$ ratio of band intensities is $c a .0 .71$, indicating that GQDs are highly crystalline and graphitic.

Figure 1c shows the UV-vis spectrum of GQDs. It can be observed that there is a dominant absorption peak centered at $220 \mathrm{~nm}$ together with a shoulder at $350 \mathrm{~nm}$, which can be attributed to $\pi-\pi^{*}$ transition of $\mathrm{C}=\mathrm{C}$ and $\mathrm{n}-\pi^{*}$ transition of $\mathrm{C}=\mathrm{O}$, respectively. Meanwhile, it can be seen that a long tail extends into a visible range. These features are similar to those of UV-vis behaviors of GQDs [26, 27]. Figure 1d presents the room temperature PL spectra of GQDs. The emission peaks centered at $540 \mathrm{~nm}$ are independent of excitation wavelengths in the 300-440 $\mathrm{nm}$ range, indicating that the surfaces of those $s p^{2}$ clusters contained in GQDs are uniform [28]. 

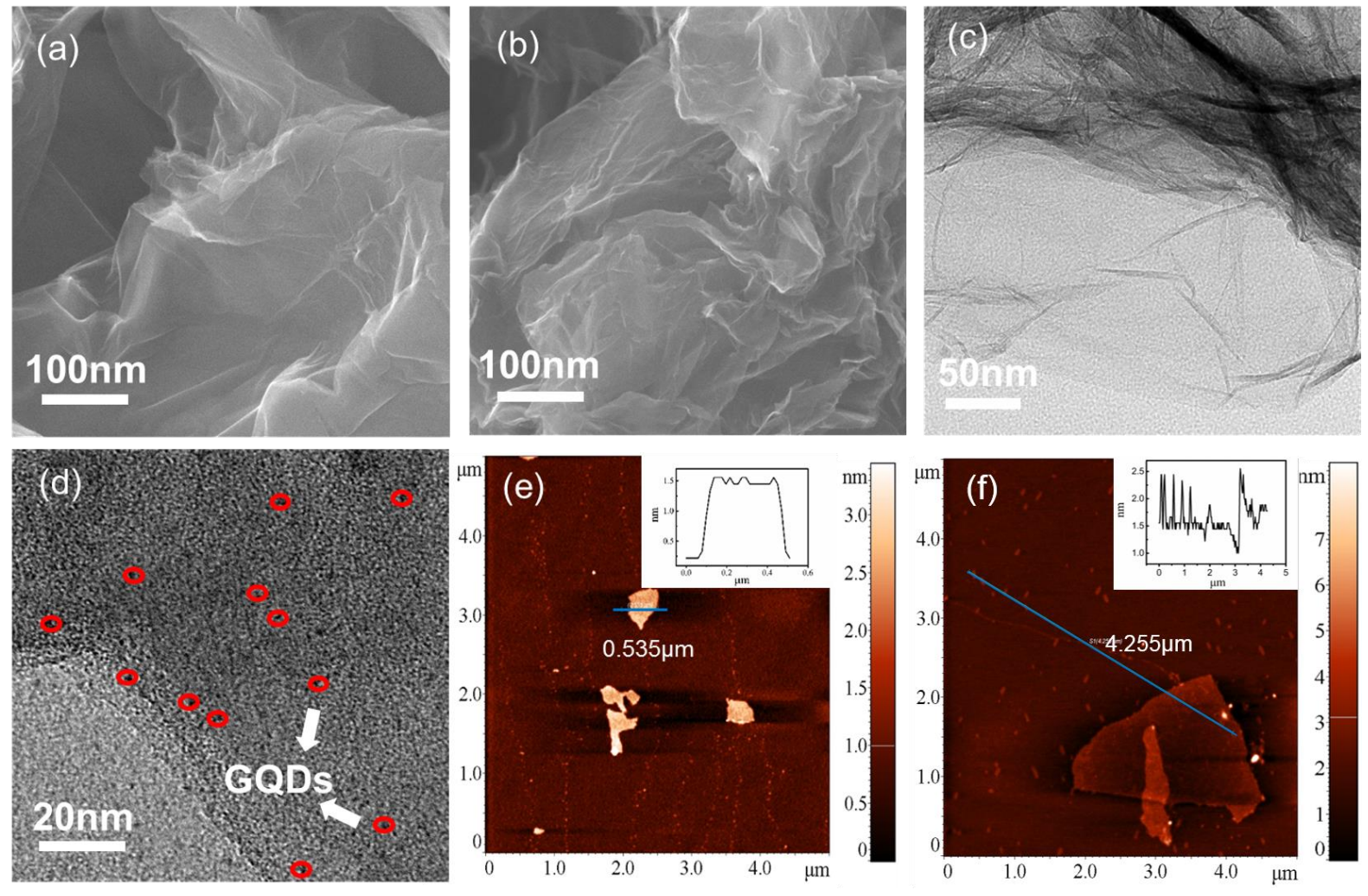

Figure 2 Characterization of rGO and rGO/GQDs: SEM images (a, b) and TEM images (c, d). AFM images of rGO (e) and rGO/GQDs (f). Insets: the height profile of the blue line section.

Figure 2a and $\mathbf{b}$ display the typical SEM images of rGO and rGO/GQDs, respectively. Compared with rGO, the composite of rGO/GQDs shows a fluffy structure with more folded edges. In Figure 2c, the crumpled rGO sheets are transparent as thin films and show the typical two-dimensional structure with folded edges. For rGO/GQDs, the rGO sheets are uniformly decorated by GQDs (red circles) as shown in Figure 2d, demonstrating that GQDs are well dispersed and combined with rGO sheets. The insertion of GQDs between the rGO sheets layers forms a sandwich-like structure. Figure 2e and $\mathbf{f}$ show the AFM images of rGO and rGO/GQDs, respectively. The AFM image of rGO exhibits that the rGO sheets have a thickness distribution of $\sim 0.3-1.5 \mathrm{~nm}$, indicating that the rGO consists of 1-4 layers of graphene. In Figure 2f, the rGO/GQDs have a thickness distribution of $~ 1-2.5 \mathrm{~nm}$, the GQDs are well dispersed and combined with rGO sheets. These results further confirm that the rGO 
sheets are uniformly decorated by GQDs. The GQDs serving as spacer insertion between the rGO sheets can reduce the agglomeration of the $\mathrm{rGO}$ and provide more access for the electron transfer within the interlayer of rGO sheets.
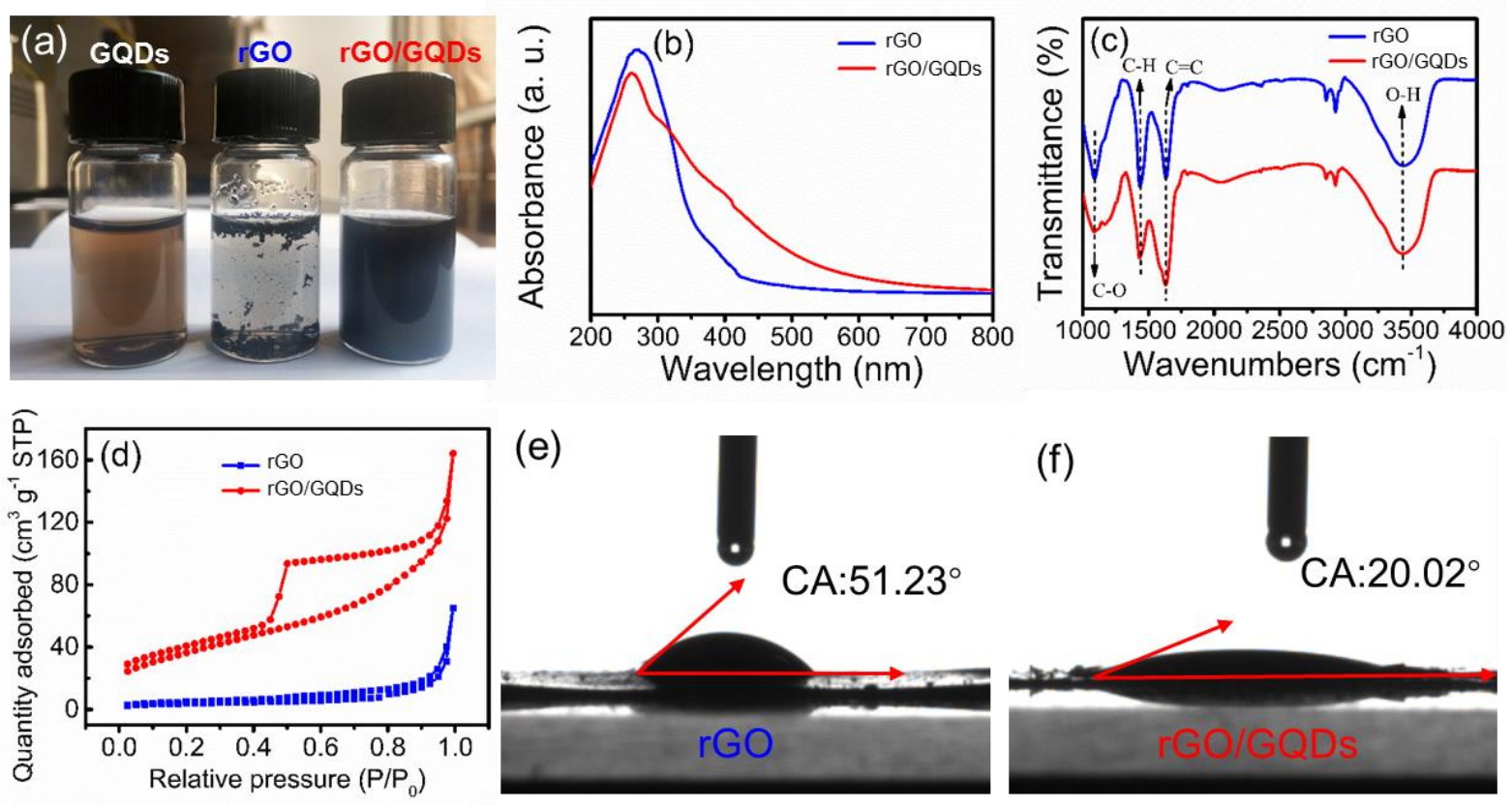

Figure 3 (a) Photographs of GQDs, rGO and rGO/GQDs aqueous solutions from left to right. UV-vis absorption spectra (b), FTIR spectra (c) and $\mathrm{N}_{2}$ adsorption-desorption isotherms (d) of the rGO and rGO/GQDs. CA test images of rGO (e) and rGO/GQDs composite (f).

Figure 3a displays the photographs of GQDs, rGO and rGO/GQDs aqueous solution, respectively. The GQDs exhibit prominent solubility in deionized water. Nevertheless, the dispersibility of rGO powder in deionized water is quite low due to its relatively high hydrophobic nature, the obvious precipitation and flotage can be observed for rGO aqueous solution. In contrast, the rGO/GQDs composite forms a homogeneous and stable suspension in aqueous solution without agglomeration, suggesting that the introduction of GQDs significantly reduces the hydrophobic rGO surface exposed to water [29]. Therefore, the rGO/GQDs can be stably dissolved in water. 
The reduction degree of $\mathrm{rGO}$ and composition change of $\mathrm{rGO} / \mathrm{GQD}$ dispersion can be monitored by UV-vis absorbance spectroscopy. In Figure $\mathbf{3 b}$, rGO exhibits a dominant absorption peak at $271 \mathrm{~nm}$, suggesting that graphene oxide has been reduced by hydrazine hydrate and rGO sheets maintain the $s p^{2}$ carbon structure during dispersing processes. There is a prominent absorption peak centered at $266 \mathrm{~nm}$ for the $\mathrm{rGO} / \mathrm{GQD}$ dispersion, which is an indication of the adsorption of GQDs on rGO sheets [30].

Figure 3c presents the FTIR spectra of rGO and rGO/GQDs, which can directly reveal the chemical bonds of rGO and rGO/GQDs. The absorption peaks at 1080, 1640, 3440 and1429 $\mathrm{cm}^{-1}$ can be attributed to the modes of $\mathrm{C}-\mathrm{O}, \mathrm{C}=\mathrm{C}, \mathrm{O}-\mathrm{H}$ stretching vibrations and $\mathrm{C}-\mathrm{H}$ bending vibration, respectively. For $\mathrm{rGO}$, the presence of $\mathrm{C}=\mathrm{C}$ bonds indicates that graphene oxide is reduced by hydrazine hydrate. Meanwhile, the presence of $\mathrm{C}-\mathrm{O}$ and $\mathrm{O}-\mathrm{H}$ bonds suggests that the oxygen-containing groups have been incompletely removed from the carbon backbone of rGO. Compared with rGO, the intensities of rGO/GQDs absorption peaks keep almost unchanged, indicating that the $s p^{2}$ carbon structure of rGO maintains unchanged during the GQDs adsorption processes. XPS measurements have been employed to further study the contents of carbon and oxygen in the rGO and rGO/GQDs quantitatively. As shown in Figure S2 (Supporting Information), two peaks at 285 and $535 \mathrm{eV}$ correspond to the $\mathrm{C} 1 \mathrm{~s}$ and $\mathrm{O} 1 \mathrm{~s}$, respectively. The content of oxygen for $\mathrm{rGO}$ and $\mathrm{rGO} / \mathrm{GQD}$ is $c a .7 .03 \%$ and $17.01 \%$, respectively. And the ratio of $\mathrm{O} / \mathrm{C}$ of $\mathrm{rGO}$ and $\mathrm{rGO} / \mathrm{GQDs}$ is 0.08 and 0.20 , respectively. Compared with $\mathrm{rGO}$, the increase of $\mathrm{O} / \mathrm{C}$ ratio for $\mathrm{rGO} / \mathrm{GQD}$ may be derived from the oxygen-containing functional groups of GQDs, which is beneficial to effectively increase the wettability between electrolyte ions and the rGO as electrode materials. As shown in Figure S3 (Supporting Information), the C1s spectra of the rGO and rGO/GQDs can be well fitted with $\mathrm{C}-\mathrm{C} / \mathrm{C}=\mathrm{C}(284.76 \mathrm{eV}), \mathrm{C}-\mathrm{O}(285.45 \mathrm{eV})$, and $\mathrm{C}=\mathrm{O}(287.12 \mathrm{eV})$ [18]. The O1s spectra can be fitted into two peaks at 531.42 and $533.16 \mathrm{eV}$, corresponding to $\mathrm{C}=\mathrm{O}$ and $\mathrm{C}-\mathrm{OH} / \mathrm{C}-\mathrm{O}-$ $\mathrm{C}$, respectively [18]. Compared with $\mathrm{rGO}$, the contents of $\mathrm{C}=\mathrm{O}$ and $\mathrm{C}-\mathrm{OH} / \mathrm{C}-\mathrm{O}-\mathrm{C}$ of 
rGO/GQDs comparatively increase, which are considered to make a contribution to the diffusion-controlled behaviors.

Figure S4 (Supporting Information) displays the Raman spectra of GO, rGO, and rGO/GQD composites. It can be seen that the D band at $1360 \mathrm{~cm}^{-1}$ is ascribed to structural defects, and $\mathrm{G}$ band at $1600 \mathrm{~cm}^{-1}$ is associated with the $s p^{2}$ carbon structure. The $\mathrm{I}_{\mathrm{D}} / \mathrm{I}_{\mathrm{G}}$ ratio of GO and $\mathrm{rGO}$ are $c a .0 .94$ and 0.91 , indicating that the reduction of GO sheets. Compared with that of rGO, the values of $\mathrm{ID}_{\mathrm{D}} / \mathrm{I}_{\mathrm{G}}$ for $\mathrm{rGO} / \mathrm{GQD}(3: 3)$ is $c a$. 0.86 , suggesting the increase of the graphitic degree and the maintain of $s p^{2}$ carbon structure during the adsorption of GQDs.

Figure 3d shows the $\mathrm{N}_{2}$ adsorption-desorption isotherms of the $\mathrm{rGO}$ and $\mathrm{rGO} / \mathrm{GQDs}$. As shown in Figure 3d, the rGO and rGO/GQDs exhibit specific surface area of $133.6 \mathrm{~m}^{2} \mathrm{~g}^{-1}$ and $155.0 \mathrm{~m}^{2} \mathrm{~g}^{-1}$, respectively. The specific surface area of rGO/GQDs is enhanced due to the anchoring of GQDs. The typical IV isotherm characteristics with a wide hysteresis loop can be observed. It should be noted that the rGO/GQDs composite has strong adsorption below the relative pressure of $P / P_{0}=0.5$, suggesting a feature of micropore filling of rGO/GQDs. Figure S5 (Supporting Information) displays the pore size distribution of rGO and rGO/GQDs, these pores with a narrow distribution range from 2 to $4 \mathrm{~nm}$, and the microspores of rGO/GQDs increases significantly after the decoration with GQDs, which can facilitate the electron transfer in the rGO/GQDs electrode.

To evaluate the wettability, the CA tests of rGO and rGO/GQDs composite are implemented, as shown in Figure 3e and $\mathbf{f}$. The $\mathrm{CA}$ of a water droplet on the rGO and rGO/GQDs composite is 51.23 and 20.02 degrees, respectively. These results demonstrate that the rGO/GQDs composite exhibits the improved wettability compared with rGO.

\subsection{Electrochemical behaviors}



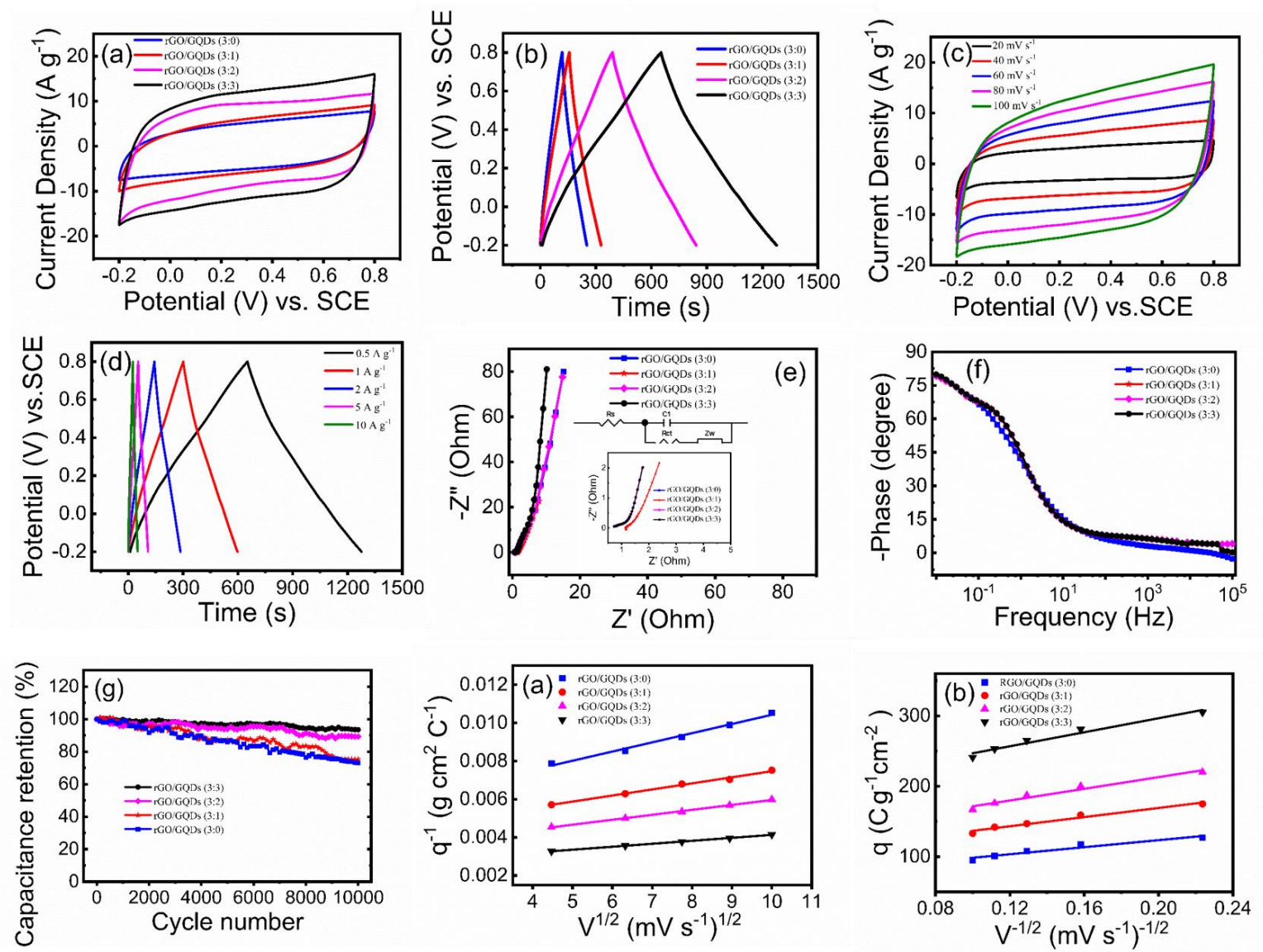

Figure 4 (a) CV and (b) GCD curves of rGO/GQDs electrodes with mass ratio=3:0, 3:1, 3:2, and 3:3 at $80 \mathrm{mV} \mathrm{s}^{-1}$ and $0.5 \mathrm{~A} \mathrm{~g}^{-1}$, respectively. (c) $\mathrm{CV}$ and (d) GCD curves of rGO/GQDs (3:3) electrode. (e) Nyquist plots of rGO/GQDs electrodes. Inset: an expanded view in the region of high frequencies and an equivalent circuit model. (f) Plot of the impedance phase angle versus the frequency for rGO/GQDs. (g) Preserved $C_{s}$ of rGO/GQDs electrode after 10000 charge/discharge cycles. Plot for the dependence of $q^{-1}$ on $v^{1 / 2}$ (h) and $q$ on $v^{-1 / 2}$ (i) for rGO/GQDs at different mass ratios.

In order to study electrochemical behaviors of rGO/GQDs composite, the electrodes are prepared by changing the mass ratio of GQDs to rGO from 3:0 to 3:3. Figure 4a displays the $\mathrm{CV}$ curves of $\mathrm{rGO} / \mathrm{GQD}$ electrodes at $80 \mathrm{mV} \mathrm{s}^{-1}$. A relatively ideal rectangular shape without obvious redox peaks can be observed, demonstrating that rGO/GQDs electrodes exhibit ideal electric double-layer capacitive behaviors [19]. With the increase of the mass ratio of 
rGO/GQDs from 3:0 to 3:3, the area enclosed by the CV curves enhances nearly two times, suggesting that the high specific capacitance can be obtained from the composites with high mass ratio. This result demonstrates that the GQDs play an important role in improving $C_{\mathrm{s}}$ of rGO. The enhanced electrochemical performance of the composite can be explained as the following: (i) The GQDs can increase the reaction contact interface of rGO/GQDs, resulting in fast ion and charge transport for the electrochemical reaction charge storage [31]. (ii) After the introduction of GQDs, the sandwich-like structure of rGO/GQDs reduces the internal resistance of the composite, leading to the enhanced specific capacitance of rGO/GQDs electrode.

Figure $4 \mathbf{b}$ shows GCD curves of $\mathrm{rGO} / \mathrm{GQD}$ electrodes at $0.5 \mathrm{~A} \mathrm{~g}^{-1}$. The triangle type shape without obvious potential drop can be obtained from all GCD curves, indicating that these electrodes have low internal resistance and good reversibility during the charge/discharge process [32]. The calculated specific capacitance is $132,181,226$, and $312 \mathrm{~F} \mathrm{~g} \mathrm{~g}^{-1}$ for the rGO/GQDs electrodes with the mass ratio of $3: 0,3: 1,3: 2$, and $3: 3$, respectively. The calculated specific capacitance at different current densities is shown in Table S1 (Supporting Information). The higher ratios than 3:3 were also prepared by changing mass ratios of GQDs to rGO from 3:4 to 3:5. The CV curves at different scan rates and GCD curves at different current densities of rGO/GQDs (3:4) and rGO/GQDs (3:5) are displayed in Figure S8. From the GCD curves, the calculated Cs of rGO/GQDs (3:4) and rGO/GQDs (3:5) electrode is 264 and $205 \mathrm{~F} \mathrm{~g}^{-1}$ at $0.5 \mathrm{~A} \mathrm{~g}^{-1}$, respectively. With the increase of rGO/GQDs mass ratio from 3:0 to $3: 3-5$,- the specific capacitance of $\mathrm{rGO/GQDs} \mathrm{increases} \mathrm{significantly,} \mathrm{a} \mathrm{significant}$ improvement of the specific capacitance can be obtained, and the maximum specific capacitance is obtained from rGO/GQDs (3:3) (312 $\left.\mathrm{F} \mathrm{g}^{-1}\right)$, which is due to that more GQDs acting as spacers provide more access for the transportation of electrons into the interlayer of rGO sheets. More GQDs will result in the reduction of $C_{s}$, which may be attributed to the lower exposed active surface of GQDs than rGO. Compared to the reported results of $C_{s}$ 
based on $\mathrm{rGO} / \mathrm{GQD}$ composite, this work shows higher $C_{\mathrm{s}}[19,20]$. And the electrochemical performance of the rGO/GQDs composite has been also compared with other graphene-based carbon composites reported recently, the results are shown in Table S2 (Supporting Information). The enhanced supercapacitive performance of rGO/GQDs is mainly attributed to the sandwich-like structure of rGO/GQDs composite, which can not only increase the electron conductivity of the electrode, but also overcome the resistance of the charge storage, and thus greatly facilitates electron transfer of the rGO/GQDs electrodes. Figure S6 (Supporting Information) displays the CV curves at different scan rates and GCD curves at different current densities of GQDs and $G \Theta$. From the GCD curves, the calculated Cs of GQDs and $\mathrm{G} O$ electrode is 41.1 and $76.7 \mathrm{~F} \mathrm{~g}^{-1}$ at $0.5 \mathrm{~A} \mathrm{~g}^{-1}$, respectively. These specific capacitance values are lower than those of rGO without the GQDs.

To further study the electrochemical properties of rGO/GQDs electrode in detail, the CV and GCD curves are measured as in Figure 4c, Figure 4d, Figure S7, and Figure S8 (Supporting Information). Figure $\mathbf{4 c}$ presents the representative $\mathrm{CV}$ curves of $\mathrm{rGO} / \mathrm{GQD}$ composite with a mass ratio of 3:3 in $1 \mathrm{M} \mathrm{H}_{2} \mathrm{SO}_{4}$ solution at different scan rates. With the increase of scan rate, CV curves remain a rectangular shape without obvious deviation, indicating excellent reversibility and fast charge transfer capability of rGO/GQDs electrode [33]. Figure 4d gives the GCD curves of $\mathrm{rGO} / \mathrm{GQDs}$ with mass ratio of 3:3 at different current densities, the triangle type shape without obvious potential drop can be observed from all the GCD curves, suggesting that the rGO/GQDs electrode has ideal capacitor behaviors and high coulombic efficiency [34]. Meanwhile, the GCD curves are symmetric during the charge and discharge cycles at different current densities, indicating the high reversibility and excellent capacitive characteristic of rGO/GQDs electrode [35]. The calculated $C_{s}$ of rGO/GQDs electrode is 312, 296, 284, 267, and $254 \mathrm{~F} \mathrm{~g} \mathrm{~g}^{-1}$ at $0.5,1,2,5$, and $10 \mathrm{~A} \mathrm{~g}^{-1}$, respectively. $C_{s}$ of $254 \mathrm{~F} \mathrm{~g}^{-1}(81.4 \%$ retention) is preserved when the current density is increased 20-fold, demonstrating the good rate capability of $\mathrm{rGO} / \mathrm{GQD}$ electrode. The excellent rate performance of rGO/GQDs 
composite can be ascribed to its unique sandwich-like structure, which provides more spaces for the rapid charge/ion transfer.

Electrochemical impedance spectrometry is an informative technique to evaluate the internal resistance of the electrodes system. The measurements of rGO/GQDs electrodes with different mass ratios are performed from $100 \mathrm{kHz}$ to $0.01 \mathrm{~Hz}$. Figure 4e displays the Nyquist plots of rGO/GQDs electrode with mass ratios of 3:0 and 3:3. It can be observed that all the plots of rGO/GQDs electrode present the straight lines in the low-frequency region. Moreover, for rGO/GQDs (3:3) electrode, it is nearly paralleled to the imaginary impedance axis, which indicates that the rGO/GQDs electrode with high mass ratio (3:3) exhibits good capacitive behavior $[22,36]$. The inset is an equivalent circuit model of the experimental data, where $R_{s}$, $R_{c t}, Z_{w}$ and $C_{1}$ is equivalent series, charge transfer resistance, Warburg impedance, and capacitance, respectively [37]. In the high-frequency region, the intersections with the real axis represent $R_{s .} R_{s}$ values of rGO/GQDs electrodes with mass ratios of 3:0 and 3:3 is $\sim 0.80$ and $0.89 \Omega$, respectively, which indicates the good contact between the graphite foil and rGO/GQDs. $R_{c t}$ value of the $\mathrm{rGO} / \mathrm{GQDs} 3: 0$ and $3: 3$ corresponds to 1.62 and $0.76 \Omega$, respectively, suggesting the excellent electrons mobility of rGO/GQDs (3:3), which can be attributed to the reduction of agglomeration among the rGO after the introduction of enough GQDs. In the Bode plot, it is well-known that the closer the phase angle approaches $90^{\circ}$ at low frequencies, the more excellent capacitor behavior of electrode material can be obtained. From the bode phase diagrams (Figure 4f), the phase angles of rGO/GQDs (3:0) and rGO/GQDs (3:3) are 79.85 and 80.10 degrees at $0.01 \mathrm{~Hz}$, respectively, which indicates an almost full double layer capacitor behavior. A further comparison of the frequency response of the rGO/GQDs (3:0) and rGO/GQDs (3:3) can be made by analyzing the relaxation time constant $\left(\tau_{0}\right)$, which is calculated by the equation of $\tau_{0}=1 / f_{0}$, in which $f_{0}$ is the frequency at the angle of $45^{\circ}$. The calculated $\tau_{0}$ of rGO/GQDs (3:0) and rGO/GQDs (3:3) are $1.35 \mathrm{~s}$ and $0.90 \mathrm{~s}$, respectively. The small relaxation time constant of the rGO/GQDs (3:3) reveals the 
high-power response of the electrode material, which further confirms the fast diffusion of electrolyte ions on the surface of rGO/GQDs electrode.

Figure $\mathbf{4 g}$ displays the preserved $C_{\mathrm{s}}$ of $\mathrm{rGO} / \mathrm{GQD}$ electrodes after 10,000 cycles at $1 \mathrm{~A} \mathrm{~g} \mathrm{~g}^{-1}$. The $C_{\mathrm{s}}$ retention is $75 \%, 80 \%, 92 \%$ and $93 \%$ for the rGO/GQDs electrodes with mass ratio of $3: 0,3: 1,3: 2$, and 3:3, respectively. This result indicates that the rGO/GQDs electrode shows very stable energy-storage performance in repetitive charge-enhanced supercapacitive performances.

In order to study the synergistic effect between rGO and GQDs for the enhanced electrochemical performances of $\mathrm{rGO} / \mathrm{GQD}$, it is necessary to quantify the relative contributions from double-layer capacitive behavior $\left(C_{d l}\right)$ and diffusion-controlled capacitance $\left(C_{d}\right)$. The total specific capacitance $\left(C_{S, T}\right)$ is the combination of $C_{d l}$ and $C_{d}$, which can be evaluated by varying the scan rates. Figure $5 \mathrm{~h}$ and i present the point diagrams of $q^{-1} v s v^{1 / 2}$ and $q^{1} v s v^{-1 / 2}$, which are obtained by linear fitting the data point of rGO/GQDs at different scan rates. The total voltammetric charge $\left(q_{T}\right)$ is calculated by extrapolation of $q_{T}$ to $v=0$ from the plot of $q^{-1} v s v^{1 / 2}$, the $q_{d l}$ can be obtained from the extrapolation of $v=\infty$ from the plot of $q v s v^{-1 / 2} . q_{p}$ is calculated from the difference between $q_{T}$ and $q_{d l}$. Furthermore, the values of $C_{S, T}, C_{d l}$, and $C_{d}$ can be obtained through charge divided by the potential window of CV. All the values are shown in Table S1 S3. The $C_{d}$ contribution is mainly attributed to the slight oxygen-containing functional groups in the rGO and GQDs, and the $C_{d l}$ contribution is related to rGO itself. With the increase of rGO/GQDs mass ratio from 3:0 to 3:3, all the electrodes show a low $C_{d}$ contribution ratio of about $30 \%$, indicating that the number of GQDs does not significantly change the $C_{d}$ contribution. By comparison, with the increase of rGO/GQDs mass ratio, the $C_{d l}$ increases significantly from 97.3 to $249.1 \mathrm{~F} \mathrm{~g}^{-1}$, suggesting that the number of GQDs effectively improves the capacitance contribution of rGO. The rGO sheets provide spaces for the adsorption of GQDs, and the adsorbed GQDs can improve the 
space utilization of rGO interlayer. Therefore, the introduction of GQDs effectively prevents aggregation and restack of rGO sheets, promoting the rGO surface exposure for sufficient contact with the electrolyte, which can provide more access for the transportation of electrons in the interlayer of rGO sheets. Consequently, the rGO/GQDs electrodes exhibit excellent supercapacitor performances by the effective synergistic effect between rGO and GQDs.
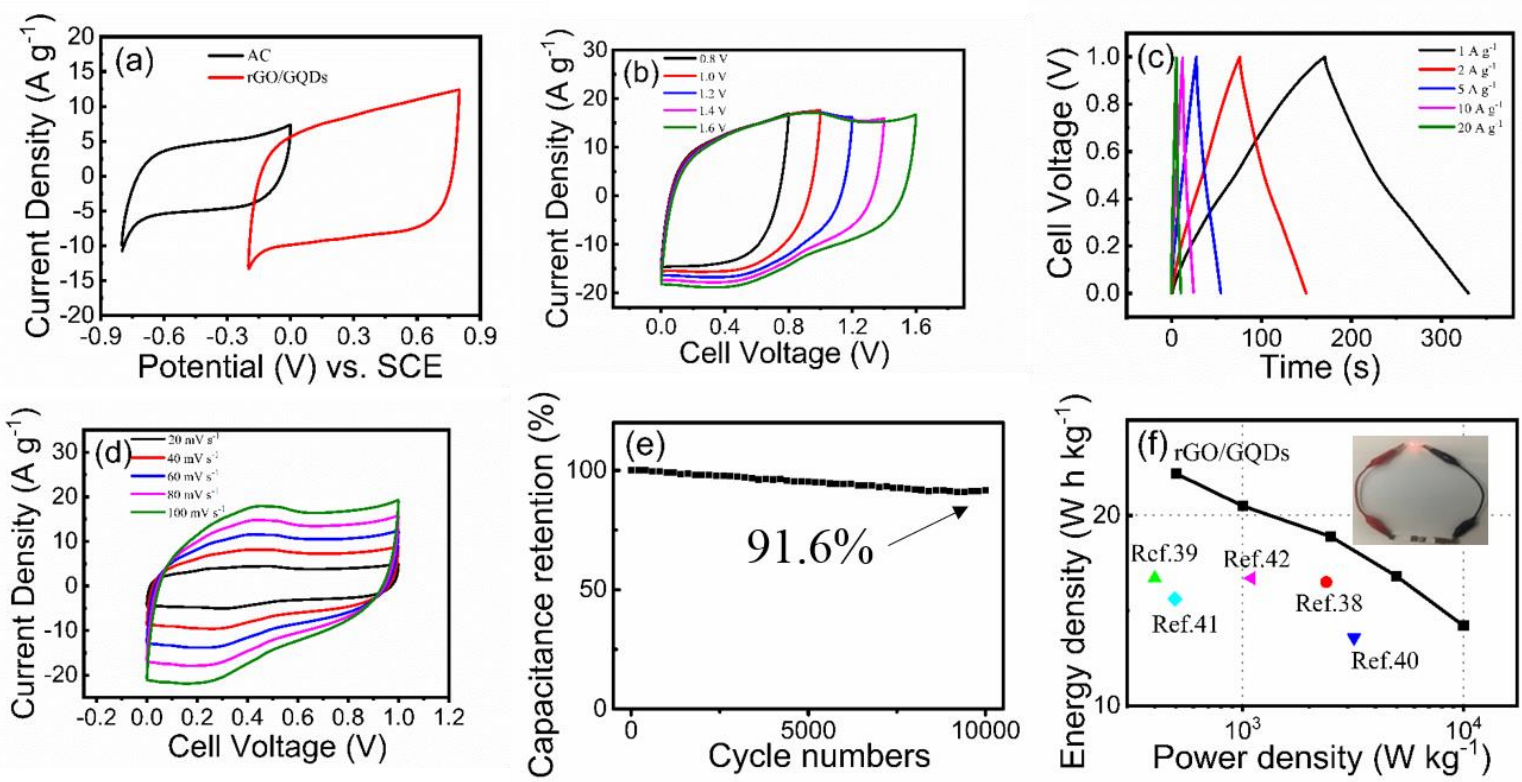

Figure 5 (a) $\mathrm{CV}$ curves of $\mathrm{AC}$ and $\mathrm{rGO} / \mathrm{GQDs}$ (3:3) electrode performed in three-electrode system at $60 \mathrm{mV} \mathrm{s}^{-1}$. (b) $\mathrm{CV}$ curves of $\mathrm{rGO} / \mathrm{GQD} / / \mathrm{AC}$ ASC performed under different potential windows at $100 \mathrm{mV} \mathrm{s}^{-1}$. (c) GCD curves of rGO/GQDs//AC ASC at different current densities. (d) $\mathrm{CV}$ curves of $\mathrm{rGO} / \mathrm{GQD} / / \mathrm{AC}$ ASC. (e) Preserved $C_{s}$ of $\mathrm{rGO} / \mathrm{GQD} / / \mathrm{AC}$ ASC at $5 \mathrm{~A} \mathrm{~g}^{-1}$. (f) Ragone plots of rGO/GQDs//AC ASC. The inset: photograph of light-emitting diode powered by two rGO/GQDs//AC ASCs.

To further investigate the supercapacitor properties of the rGO/GQDs composite in practical applications, the rGO/GQDs//AC ASC is fabricated. Figure 5a presents the CV curves of AC and $\mathrm{rGO} / \mathrm{GQDs}(3: 3)$ electrode at $60 \mathrm{mV} \mathrm{s}^{-1}$. AC and $\mathrm{rGO} / \mathrm{GQDs}$ electrodes work in the potential range from -0.8 to $0 \mathrm{~V}$ and from -0.2 to $0.8 \mathrm{~V}$, respectively. It can be concluded that the ASC potential extends up to $1.6 \mathrm{~V}$. Figure $\mathbf{5 b}$ shows $\mathrm{CV}$ curves of $\mathrm{rGO} / \mathrm{GQD}$ //AC ASC 
performed with diverse working potential at $100 \mathrm{mV} \mathrm{s}^{-1}$. The ideal rectangular shape without obvious peaks can be obtained from all the CV curves, suggesting a wide potential window and good reversibility. Figure 5c displays the GCD curves of the ASC at various current densities. The linear slope and triangle shape can be observed for all the GCD curves, suggesting the good reversibility and good rate capability of the supercapacitor. Figure S9 (Supporting Information) displays the GCD curves of the ASC with the potential window increasing from 0.8 to $1.6 \mathrm{~V}$. The GCD curves are symmetric at work potential as high as 0 1.6 $\mathrm{V}$ at $2 \mathrm{~A} \mathrm{~g} \mathrm{~g}^{-1}$, demonstrating the ideal capacitive behavior and high reversibility of the rGO/GQDs//AC ASC. Figure 5d displays the typical CV curves of the ASC. All CV curves show an ideal rectangular shape, indicating the fast charge transport and good capacitive characteristics of the supercapacitor. Figure 5e shows the cycling performance of the ASC. It can be seen that $91.6 \%$ of $C_{s}$ retention at $5 \mathrm{~A} \mathrm{~g}^{-1}$ can be obtained after 10000 cycles. The good cycling stability can be attributed to the reduction of agglomeration among the rGO after the introduction of GQDs. Figure S10 (supporting information) shows the Nyquist plots for $\mathrm{rGO} / \mathrm{GQD} / / \mathrm{AC}$ ASC, the inset is an expanded view in the region of high frequencies. It can be observed that the plots of $\mathrm{rGO} / \mathrm{GQD} / / \mathrm{AC}$ ASC present the straight lines in the lowfrequency region, which indicates that the $\mathrm{rGO} / \mathrm{GQD} / / \mathrm{AC}$ ASC exhibits good capacitive behavior. Figure $\mathbf{5 f}$ displays the Ragone plots of $\mathrm{rGO} / \mathrm{GQDs} / \mathrm{AC}$ ASC, together with the illuminated light-emitting diodes powered by two rGO/GQDs//AC ASCs, as shown in the inset of Figure 5f. Two rGO/GQD//AC ASC can light up light-emitting diodes for 20 seconds. The energy and power density of rGO/GQDs//AC ASC are $22.2 \mathrm{~W} \mathrm{~h} \mathrm{~kg}$-1 and $500 \mathrm{~W} \mathrm{~kg}^{-1}$, respectively. These results are higher than the previous reports, such as PVA-GQD$\mathrm{Co}_{3} \mathrm{O}_{4} / \mathrm{PEDOT}$ composite(16.6 $\mathrm{W} \mathrm{h} \mathrm{kg}^{-1}$ at $\left.2396.99 \mathrm{~W} \mathrm{~kg}^{-1}\right)$ [38], 3D graphene/polymer composite (16.5 $\mathrm{W} \mathrm{h} \mathrm{kg}^{-1}$ at $401.2 \mathrm{~W} \mathrm{~kg}^{-1}$ ) [39], $\mathrm{MnO}_{2} / \mathrm{MnCO}_{3} / \mathrm{rGO}$ composite (13.57 $\mathrm{W} \mathrm{h}$ $\mathrm{kg}^{-1}$ at $3200 \mathrm{~W} \mathrm{~kg}^{-1}$ ) [40], PVA-GQD/PEDOT composite (15.62 $\mathrm{W} \mathrm{h} \mathrm{kg}^{-1}$ at $495 \mathrm{~W} \mathrm{~kg}^{-1}$ ) [41], and G-PNF composite (16.7 $\mathrm{W} \mathrm{h} \mathrm{kg}^{-1}$ at $\left.1100 \mathrm{~W} \mathrm{~kg}^{-1}\right)$ [42]. Thus, these results of our work 
demonstrate that the rGO/GQDs composite could be a promising material in electrochemical capacitors for energy storage applications.

\section{Conclusions}

In summary, the rGO sheets decorated with GQDs with an average size of $\sim 3.6 \mathrm{~nm}$ have been fabricated by a facile low-power ultrasonic method. The surface engineering strategy proposed here contributes to a higher specific capacity, an excellent rate capability and stability for both electrode and whole device evaluation. It also suggests that the $C_{d l}$ of rGO materials can be improved effectively through the synergistic effect between rGO and GQDs, as GQDs effectively prevent the aggregation of rGO sheets, and improve the wettability of rGO surface. These changes provide more efficient pathway for the transportation of electron among the interlayers of rGO sheets. This work highlights the importance of surface engineering strategies not only for supercapacitor electrodes but also a general optimization process for other energy storage materials.

\section{Acknowledgments}

This work was supported by the NSFC (Nos. 11504229, 51602193), Shanghai "Chen Guang" project (16CG63), the Talent Program of Shanghai University of Engineering Science, ESI Program of Shanghai University of Engineering Science (ESI201809, ESI201802) and the STFC Batteries Network (ST/R006873/1).

\section{References}

[1] C. N. R. Rao, K. Gopalakrishnan, A. Govindaraj, Nano Today. 2014, 9, 324.

[2] C. Zhong, Y. Deng, W. Hu, J. Qiao, L. Zhang, J. Zhang, Chem. Soc. Rev. 2015, 44,7484.

[3] X. Liu, S. Zou, K. Liu, C. Lv, Z. Wu, Y. Yin, T. Liang, Z. Xie, J. Power Sources, 2018, 384, 214.

[4] C. Yang, N. Hu, W. Wang, B. Cao, J. Power Sources, 2018, 399, 115.

[5] M. F. El-Kady, V. Strong, S. Dubin, R. B. Kaner, Science, 2012, 335,1326. 
[6] R. Z. Li, R. Peng, K. D. Kihm, S. Bai, D. Bridges, U. Tumuluri, Z. Wu, T. Zhang, G. Compagnini, Z. Feng, Energy. Environ. Sci., 2016, 9, 1458.

[7] Y. Wang, W. Lai, N. Wang, Z. Jiang, X. Wang, P. Zou, Z. Lin, H. J. Fan, F. Kang, C. P. Wong, Energy. Environ. Sci., 2017, 10, 941.

[8] F. Zhang, Y. Tang, H. Liu, H. Ji, C. Jiang, J. Zhang, X. Zhang, C. S. Lee, ACS Appl. Mater. Interfaces, 2016, 8, 4691.

[9] M. Khandelwal, Y. Li, A. Molla, S. Hyun Hur, J. Suk Chung, Chem. Eng. J., 2017, 330, 965.

[10] Q. Wang, J. Yan, Z. Fan, Energy. Environ. Sci., 2016, 9, 729.

[11] K. Lee, Y. Yoon, Y. Cho, S. M. Lee, Y. Shin, H. Lee, H. Lee, ACS Nano, 2016, 10, 6799.

[12] M. Amjadi, J. L. Manzoori, T. Hallaj, , J. Lumin., 2014, 153, 73.

[13] Y. Du, S. Guo, Nanoscale, 2016, 8, 2532.

[14] A. Bayat, E. Saievar-Iranizad, J. Lumin., 2017, 192, 180.

[15] D. Shen, W. Zhang, F. Xie, Y. Li, A. Abate, M. Wei, J. Power Sources, 2018, 402, 320.

[16] W.-W. Liu, Y.-Q. Feng, X.-B. Yan, J.-T. Chen, Q.-J. Xue, Adv. Funct. Mater., 2013, 23, 4111-4122.

[17] S. Zhang, L. Sui, H. Dong, W. He, L. Dong, L. Yu, ACS Appl. Mater. Inter., 2018, 10,12983 .

[18] M. Zheng, X. Zhao, M. Li, H. Dong, Y. Liu, H. Hu, Y. Cai, Y. Liang, Y. Xiao, Chemsuschem, 2017, 10, 2626.

[19] A. K. Samantara, S. C. Sahu, A. Ghosh, B. K. Jena, J. Mater. Chem. A, 2015, 3, 16961.

[20] Y. Q. Dang, S. Z. Ren, G. Liu, J. Cai, Y. Zhang, J. Qiu, Nanomaterials-Basel, 2016, 6, 212.

[21] S. Stankovich, R. D. Piner, S. T. Nguyen, R. S. Ruoff, Carbon, 2006, 44, 3342.

[22] M. D. Stoller, S. Park, Y. Zhu, J. An, R. S. Ruoff, Nano Lett., 2008, 8, 3498.

[23] G. L. Wang, X. Fang, X. M. Wu, X. L. Hu, Z. J. Li, Biosens. Bioelectron., 2016, 81, 214.

[24] X. Jian, J.-g. Li, H.-m. Yang, L.-1. Cao, E.-h. Zhang, Z.-h. Liang, Carbon, 2017, 114, 533. 
[25] M. Li, C. Yu, C. Hu, W. Yang, C. Zhao, S. Wang, M. Zhang, J. Zhao, X. Wang, J. Qiu, Chem. Eng. J., 2017, 320, 570.

[26] K. Hola, M. Sudolska, S. Kalytchuk, D. Nachtigallova, A. L. Rogach, M. Otyepka, R. Zboril, ACS Nano, 2017, 11,12402.

[27] H. Liu, W. Na, Z. Liu, X. Chen, X. Su, Biosens. Bioelectron., 2017, 92, 229.

[28] Y. Dong, J. Shao, C. Chen, H. Li, R. Wang, Y. Chi, X. Lin, G. Chen, Carbon, 2012, 50, 4738.

[29] B. Unnikrishnan, C.-W. Wu, I. W. P. Chen, H.-T. Chang, C.-H. Lin, C.-C. Huang, ACS Sustain. Chem. Eng., 2016, 4,3008.

[30] P. He, J. Sun, S. Tian, S. Yang, S. Ding, G. Ding, X. Xie, M. Jiang, Chem. Mater., 2014, 27,218 .

[31] D. I. Abouelamaiem, G. He, I. Parkin, T. P. Neville, A. B. Jorge, S. Ji, R. Wang, M.-M. Titirici, P. R. Shearing and D. J. L. Brett, Sustain. Energy \& Fuels, 2018, 2, 772.

[32] G. He, X. Han, R. Zou, T. Zhao, Z. Weng, S. Ho-Kimura, Y. Lu, H. Wang, Z. X. Guo and I. P. Parkin, Adv. Funct. Mater., 2017, 27,1604903.

[33] G. Chen, T. Chen, K. Hou, W. Ma, M. Tebyetekerwa, Y. Cheng, W. Weng, M. Zhu, Robust, Carbon, 2018, 127,218.

[34] A. B. Ganganboina, A. D. Chowdhury, R.-a. Electrochim. Acta, 2017, 245, 912.

[35] R. Lin, Z. Li, D. I. Abou El Amaiem, B. Zhang, D. J. L. Brett, G. He and I. P. Parkin, J. Mater. Chem. A, 2017, 5, 25545.

[36] Z. Zhao, Y. Xie, J. Power Sources, 2017, 337, 54.

[37] B. Liu, M. Yang, H. Chen, Y. Liu, D. Yang, H. Li, J. Power Sources, 2018, 397, 1.

[38] S. N. J. S. Z. Abidin, M. S. Mamat, S. A. Rasyid, Z. Zainal, Y. Sulaiman, Electrochim. Acta, 2017, 261, 548.

[39] F. Barzegar, A. Bello, O. O. Fashedemi, J. K. Dangbegnon, D. Y. Momodu, F. Taghizadeh, N. Manyala, Electrochim. Acta, 2015, 180, 442.

[40] Y. Liu, D. He, H. Wu, J. Duan, Y. Zhang, Electrochim. Acta, 2015,164,154. 
[41] S. Z. A. Jannah, Shariffah Nur, S. Mamat, S. Abdul Rasyid, Z. Zainal, Y. Sulaiman, J. Polym. Sci. Pol. Chem., 2018, 56,50.

[42] Q. Wu, Y. Xu, Z. Yao, A. Liu, G. Shi, ACS Nano, 2010, 4, 1963. 